\title{
A SEARCH FOR DARK MATTER IN THE HALOS OF LENSING GALAXIES USING VLBI
}

\author{
M.A. GARRETT ${ }^{1}$, S. NAIR ${ }^{1}$, R.W. PORCAS ${ }^{2}$ \& A.R. PATNAIK ${ }^{2}$ \\ ${ }^{1}$ NRAL, Jodrell Bank, UK; ${ }^{2}$ MPIfR, Bonn, Germany
}

\section{Introduction}

Baryonic Dark Matter (BDM) candidates are segregated into two main mass ranges: (i) sub-solar mass dwarf stars (MACHOS) and (ii) $\sim 10^{4}-$ $10^{6} M_{\odot}$ Very Massive Objects (VMOs). The lower mass range has been the target of the various micro-lensing programs but the first, tentative conclusions (see Stubbs et al. these proceedings) seem to suggest that MACHOs are unlikely to provide the bulk of the dark matter in the galactic halo. Meanwhile the upper mass range $\left(10^{4}-10^{6} M_{\odot}\right)$ remains largely unexplored. However, Wambsganss \& Paczynski 1992 (hereafter WP92), have shown that this mass range is perfectly tuned to a straightforward and direct test: gravitational milli-lensing of macro-lensed images (Fig 1).

\section{High resolution VLBI Observations of Resolved Lens Systems}

We have recently applied the method of WP92 to $1.6 \mathrm{GHz}$ Global VLBI observations of $0957+561 \mathrm{~A}, \mathrm{~B}$ (Garrett et al. 1994). We conclude that black holes of mass $m \geq 3 \times 10^{6} \mathrm{M}_{\odot}$ do not form a large proportion (>10\%) of the DM in the halo of the lens galaxy. However, in order to to probe the more interesting mass range of $10^{4}-10^{6} M_{\odot}$ we have embarked on a series of high frequency, sub-mas resolution observations of several radio lens systems. Here we present preliminary sub-mas images of 1830-211 (see Fig 1). The maps are difficult to interpret. While " $B$ " exhibits a 15 mas long jet to the northwest " $A$ " is essentially unresolved. It is not clear how the two images can look quite so different without resorting to contrived relative magnification matrices. Further observations, separated by the image time delay are planned. 


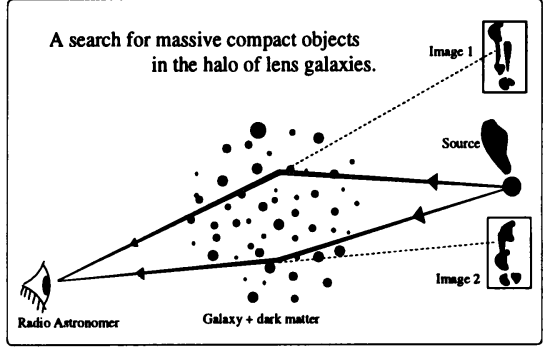

\section{$1830-211 B$}

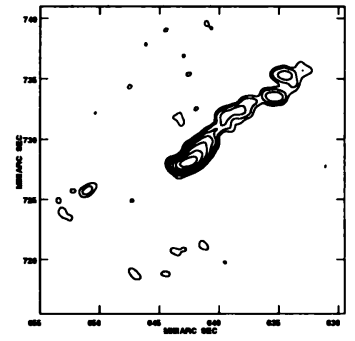

$1830-211 \mathrm{~A}$

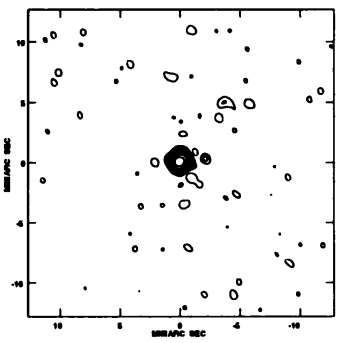

Figure 1. Left: The presence of massive compact objects in the halo of the lens galaxy will produce distortions (e.g. kinks, bends, rings, holes, faint multiple images) which will be peculiar to each image (since the line-of-sight is different for each image). Right: $15 \mathrm{GHz}$ VLBA 0.7 mas resolution maps of $1830-211 \mathrm{~A}, \mathrm{~B}$.

\section{Potential Problems}

While the method suggested by WP92 is in principle straightforward we anticipate three potential problems:

- significant changes (e.g. $\geq$ beam-size) in the images radio structure on a time scale $\sim$ time delay

- uncooperative relative magnifications - the case in which the lensed images cannot be easily compared

- biasing of the distorted images towards smoothness during the hybrid mapping process

One can overcome the first two of these problems by studying systems which exhibit low variability and for which the time delays are long (e.g. $0957+561$ ). We have recently investigated the third problem by simulating realistic visibility data "corrupted" by isolated milli-lensing events; we are satisfied that even subtle distortions can be reliably reconstructed using the standard hybrid mapping techniques.

\section{Summary}

We conclude that the prospects for the detection of uncorrelated distortions in lensed images remain good. We also note that with the enhanced resolution of the forthcoming Space VLBI programs, detection of compact masses $\geq 10^{3} M_{\odot}$ may be possible.

\section{References}

Garrett et al., 1994, MNRAS, 270, 457

Wambsganss, J., \& Paczynski, B., 1992, ApJL, 397, L1 